Seigneuret, M., \& Devaux, P. F. (1984) Proc. Natl. Acad. Sci. U.S.A. 81, 3751-3755.

Sleight, R. G., \& Pagano, R. E. (1984) J. Cell Biol. 99, 742-751.

Steck, T. L. (1974) Methods Membr. Biol. 2, 245-281.

Steck, T. L., \& Yu, J. (1973) J. Supramol. Struct. 1, 220-232.

Struck, D. K., \& Pagano, R. E. (1980) J. Biol. Chem. 255, 5404-5410.

Sune, A., Bette-Bobillo, P., Bienvenue, A., Fellmann, P., \& Devaux, P. F. (1987) Biochemistry 26, 2972-2978.
Sutherland, J. A., Turner, A. R., Mannoni, P., McGann, L. E., \& Ture, J. M. (1986) J. Biol. Response Modif. 5, 250-262.

Tilley, L., Cribier, S., Roelofsen, B., Op den Kamp, J. A. F., \& Van Deenen, L. L. M. (1986) FEBS Lett. 194, 21-27.

Verkleij, A. J., Zwaal, R. F. A., Roelofsen, B., Comfurius, P., Kastelijn, D., \& Van Deenen, L. L. M. (1973) Biochim. Biophys. Acta 323, 178-193.

Zachowski, A., Hermann, A., Paraf, A., \& Devaux, P. F. (1987) Biochim. Biophys. Acta 897, 197-200.

\title{
Spin-Label ESR Studies on the Interaction of Bovine Spinal Cord Myelin Basic Protein with Dimyristoylphosphatidylglycerol Dispersions ${ }^{\dagger}$
}

\author{
M. B. Sankaram, ${ }^{\ddagger}$ P. J. Brophy, ${ }^{\S}$ and D. Marsh ${ }^{*, \ddagger}$ \\ Max-Planck-Institut für biophysikalische Chemie, Abteilung Spektroskopie, Postfach 2841, D-3400 Göttingen, \\ Federal Republic of Germany, and Department of Biological Science, University of Stirling, Stirling FK9 4LA, \\ United Kingdom \\ Received November 2, 1988; Revised Manuscript Received July 24, 1989
}

\begin{abstract}
Electron spin resonance (ESR) spectroscopy and chemical binding assays were used to study the interaction of bovine spinal cord myelin basic protein (MBP) with dimyristoylphosphatidylglycerol (DMPG) membranes. Increasing binding of MBP to DMPG bilayers resulted in an increasing motional restriction of PG spin-labeled at the C-5 atom position in the acyl chain, up to a maximum degree of association of 1 MBP molecule per 36 lipid molecules. ESR spectra of PG spin-labels labeled at other positions in the $s n-2$ chain showed a similar motional restriction, while still preserving the chain flexibility gradient characteristic of fluid lipid bilayers. In addition, labels at the C-12 and C-14 atom positions gave twocomponent spectra, suggesting a partial hydrophobic penetration of the MBP into the bilayer. Spectral subtractions were used to quantitate the membrane penetration in terms of the stoichiometry of the lipid-protein complexes. Approximately $50 \%$ of the spin-labeled lipid chains were directly affected at saturation protein binding. The salt and $\mathrm{pH}$ dependence of the ESR spectra and of the protein binding demonstrated that electrostatic interaction of the basic residues of the MBP with the PG headgroups is necessary for an effective association of the MBP with phospholipid bilayers. Binding of the protein, and concomitant perturbation of the lipid chain mobility, was reduced as the ionic strength increased, until at salt concentrations above $1 \mathrm{M} \mathrm{NaCl}$ the protein was no longer bound. The binding and ESR spectral perturbation also decreased as the protein charge was reduced by $\mathrm{pH}$ titration to above the $\mathrm{p} I$ of the protein at approximately $\mathrm{pH} 10$. The obligatory electrostatic requirement was further evidenced by the strongly reduced binding of MBP to dimyristoylphosphatidylcholine bilayers compared with that to DMPG bilayers.
\end{abstract}

$T_{\text {he }}$ extrinsic men extrinsic membrane protein which is located in the interbilayer spaces of the multilamellar structures formed by the oligodendroglial plasma membranes (Kies et al., 1958; Laatsch et al., 1962). This protein represents $30 \%$ of the total protein present in central nervous system myelin [see Braun (1984)] and is the factor responsible for the induction of experimental allergic encephalomyelitis (Eylar et al., 1970). The molecular mechanism of this pathological activity is not precisely known, but the location of the protein on the cytoplasmic side of the myelin membrane and its binding to the membrane surface suggest that the MBP stabilizes the multilamellar compact structure by joining the apposed surfaces of the myelin plasma membranes.

\footnotetext{
${ }^{+}$M.B.S. is the recipient of a fellowship from the Alexander von Humboldt Foundation.

${ }^{\ddagger}$ Max-Planck-Institut für biophysikalische Chemie.

University of Stirling.
}

MBP binds strongly to acidic lipids (Palmer \& Dawson, 1969). The molecular weight of the protein is $18.4 \mathrm{~K}$, and, together with the proteolipid protein of molecular weight $25 \mathrm{~K}$, it constitutes approximately $80 \%$ by weight of the total protein in myelin [see Boggs and Moscarello (1978a) and Boggs et al. (1982a)]. Several physicochemical investigations on the structure of the MBP have shown that the protein is devoid of tertiary structure in aqueous solutions (Eylar \& Thompson, 1969; Chao \& Einstein, 1970; Palmer \& Dawson, 1969) but assumes highly ordered conformations in organic solvent mixtures (Liebes et al., 1975; Stone et al., 1985) and upon

\footnotetext{
1 Abbreviations: DMPG, 1,2-dimyristoyl-sn-glycero-3-phosphoglycerol; DMPC, 1,2-dimyristoyl-sn-glycero-3-phosphocholine; DPPG, 1,2-dipalmitoyl-sn-glycero-3-phosphoglycerol; ESR, electron spin resonance; MBP, bovine spinal cord myelin basic protein; $n$-PGSL, 1 -acyl2 -[ $n$-(4,4-dimethyloxazolidine- $N$-oxyl)stearoyl]-sn-glycero-3-phosphoglycerol; Tris, tris(hydroxymethyl)aminomethane; EDTA, ethylenediaminetetraacetic acid.
} 
binding to lipid bilayers (Surewicz et al., 1987).

The interactions of membrane proteins with lipid bilayers are principally hydrophobic and electrostatic in nature. Extrinsic proteins like the MBP, which are readily dissociable from membranes in high-salt media, are associated with membranes primarily by electrostatic interactions between the lipid headgroups and amino acid side chains. Integral proteins, on the other hand, interact hydrophobically with the membrane by crossing the bilayer one or several times. The electrostatic interactions of these proteins with charged lipids are weakened in high salt [see, e.g., Horvāth et al. (1988) and Marsh (1985)], but this does not result in dissociation of the protein from the membrane. An interesting question, in this context, is whether the extrinsic proteins interact with membranes also hydrophobically. Earlier studies on the MBP suggest that this protein penetrates into membranes [Papahadjopoulos et al., 1975; also see Boggs and Moscarello (1978a) and Boggs et al. (1982a)].

Spin-label electron spin resonance (ESR) spectroscopy has been used previously to demonstrate a motional restriction of lipids upon incorporation of hydrophobic integral membrane proteins into membranes [see, e.g., Marsh $(1981,1982)]$. This method employs lipid probes nitroxide spin-labeled at several positions along the acyl chains which are introduced into lipid-protein recombinants to quantitate lipid-protein interactions in terms of lipid-protein stoichiometry and lipid specificity. The results of these studies on a number of integral membrane proteins of diverse origin, reconstituted both into natural lipid extracts from the corresponding membranes and into synthetic lipid bilayers, are consistent with the notion of two motionally distinct lipid populations (Marsh \& Watts, 1982; Marsh, 1983, 1985). One population is motionally restricted and forms a boundary layer interacting with the intramembranous surface of the protein, and the other population represents the fluid lipid bilayer. The data obtained on lipid-protein recombinants using such spin-label ESR methods are in accordance with the molecular size of the proteins in question (Marsh, 1985).

We report here our results on the interaction of the MBP with membranes formed from the acidic lipid dimyristoylphosphatidylglycerol (DMPG), studied by spin-label ESR spectroscopy and chemical binding studies. Particular attention is given to the electrostatic and hydrophobic components of the association and the influence on the lipid chain mobility. A combination of both types of interaction may be necessary for the stability of the myelin sheath.

\section{Materials ANd Methods}

Materials. DMPG was prepared from dimyristoylphosphatidylcholine (DMPC) (Fluka, Buchs, Switzerland) by phospholipase D (Boehringer-Mannheim, West Germany) catalyzed transphosphatidylation (Comfurius \& Zwaal, 1977). Positional isomers, $n$-PGSL, of spin-labeled phosphatidylglycerol were synthesized as described in Marsh and Watts (1982). Egg phosphatidylcholine (Lipid Products, S. Nutfield, U.K.) was used for the synthesis. Therefore, the $s n$ - 1 chains are principally palmitoyl. The spin-labels were stored at -20 ${ }^{\circ} \mathrm{C}$ in $\mathrm{CH}_{2} \mathrm{Cl}_{2}$ at a concentration of $1 \mathrm{mg} / \mathrm{mL}$.

Protein Isolation. Bovine spinal cord was obtained fresh from the slaughterhouse and frozen immediately in liquid $\mathrm{N}_{2}$. Myelin basic protein was extracted and purified as described by Diebler et al. (1972). The protein was further purified by chromatography on a Sephadex G-75 Superfine column $(3 \times$ $90 \mathrm{~cm})$ equilibrated with $10 \mathrm{mM} \mathrm{HCl}$. The protein $\left(M_{\mathrm{r}}\right.$ 18400 ) was pure as judged by polyacrylamide gel electrophoresis in sodium dodecyl sulfate (Laemmli, 1970). Protein concentrations were measured spectrophotometrically by using a value for the extinction of $E_{1 \%}^{276.4 \mathrm{~nm}}=5.64$ (Liebes et al., 1975).

Sample Preparation. Appropriate amounts of DMPG (1 $\mathrm{mg}$ ) and $n$-PGSL ( $1 \mathrm{~mol} \%$ ) were dissolved in $\mathrm{CH}_{2} \mathrm{Cl}_{2}$. A thin film was formed by evaporating the solvent under a stream of $\mathrm{N}_{2}$ gas and drying under vacuum in a desiccator for at least $3 \mathrm{~h}$. The thin films were hydrated by adding $50 \mu \mathrm{L}$ of buffer (10 $\mathrm{mM}$ Tris, $10 \mathrm{mM} \mathrm{NaCl}$, and $0.1 \mathrm{mM}$ EDTA, $\mathrm{pH} \mathrm{8.0)}$ ) and gentle mixing. To the lipid dispersion thus obtained was added the required amount of MBP solution in the same buffer. The lipid-protein complexes precipitated instantaneously. Alternatively, the dry lipid films were hydrated directly with the MBP solution. Complexation of the protein with DMPG was complete, irrespective of the method of reconstitution employed. Recombinants at high lipid:protein ratios were pelleted at $30000 \mathrm{~g}$ in a $65 \mathrm{Ti}$ rotor, whereas recombinants with a saturating amount of MBP were centrifuged on a bench centrifuge at $3000 \mathrm{rpm}$. The recombinant pellet was washed with buffer and then transferred to a $100-\mu \mathrm{L}$ capillary for ESR spectroscopy. The samples were dissolved, after the ESR experiments, in $1 \mathrm{~N} \mathrm{NaOH}$ solution to estimate the lipid and protein contents according to Eibl and Lands (1969) and Lowry et al. (1951), respectively. Samples for the salt dependence experiments were prepared as described above in $\mathrm{pH}$ 8.0 buffers containing $10 \mathrm{mM}$ Tris, $0.1 \mathrm{mM}$ EDTA, and varying concentrations of $\mathrm{NaCl}$ in the range $0-2 \mathrm{M}$. In all cases, an excess of MBP solution (2.5 mg of protein $/ \mathrm{mg}$ of lipid) was added to ensure saturation binding. $\mathrm{pH}$ titration experiments were performed in appropriate buffer solutions of constant ionic strength, $I=0.02$.

ESR Spectroscopy. ESR spectra were recorded on a Varian E-12 Century Line 9-GHz spectrometer equipped with a nitrogen gas flow temperature regulation system. The sealed capillaries (1-mm diameter) were placed in a quartz tube containing silicone oil for thermal stability. A PDP 11/10 dedicated computer system with interactive graphics was used for digital data collection and for data processing. Spectral subtractions were performed as previously described (Marsh, 1982). Effective order parameters were calculated from the expression:

$$
S_{\text {eff }}=\frac{A_{\|}-A_{\perp}}{A_{z z}-1 / 2\left(A_{x x}+A_{y y}\right)} \frac{a_{0}^{\prime}}{a_{0}}
$$

where $2 A_{\|}$is the maximum hyperfine splitting $\left(2 A_{\max }\right)$ and $A_{\perp}$ is obtained from the relation

$A_{\perp}(G)=A_{\min }(G)+1.4\left[1-\frac{A_{\|}-A_{\min }}{A_{z z}-1 / 2\left(A_{x x}+A_{y y}\right)}\right]$

where $2 A_{\min }$ is the inner hyperfine splitting and $a_{0}$ is the effective isotropic hyperfine splitting constant given by

$$
a_{0}=1 / 3\left(A_{\|}+2 A_{\perp}\right)
$$

$a_{0}^{\prime}$ is the effective isotropic hyperfine splitting constant in the single-crystal environment in which the hyperfine tensor was determined, and is given by

$$
a_{0}^{\prime}=1 / 3\left(A_{x x}+A_{y y}+A_{z z}\right)
$$

$A_{x x}, A_{y y}$, and $A_{z z}$ being the principal values of the hyperfine tensor for doxylpropane (Jost et al., 1971). Detailed ESR line-shape simulations have shown that the spectra of lipid spin-labels contain important contributions from slow molecular motions (Lange et al., 1985). Therefore, the effective order parameters calculated by using eq 1 , which assumes the fast motional limit, can only be considered as apparent values 


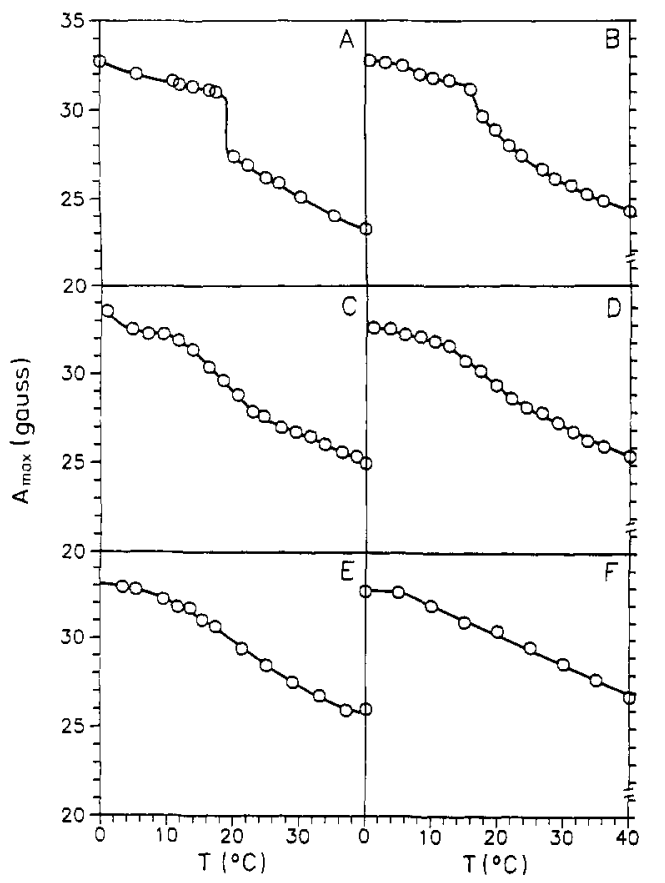

FIGURE 1: Temperature dependence of the maximum outer hyperfine splitting, $A_{\max }$, of the 5-PGSL phosphatidylglycerol spin-label in DMPG dispersions with (A) $0: 1$, (B) $0.2: 1$, (C) $0.5: 1$, (D) $0.75: 1$, (E) $1: 1$, and $(F) 2.5: 1 \mathrm{w} / \mathrm{w}$ added $\mathrm{MBP}$ in $10 \mathrm{mM}$ Tris, $10 \mathrm{mM} \mathrm{NaCl}$, and $0.1 \mathrm{mM}$ EDTA at $\mathrm{pH} 8.0$.

but are nonetheless useful for investigating the effects of protein binding.

\section{RESULTS}

Binding Curve. The myelin basic protein binds strongly to dimyristoylphosphatidylglycerol dispersions at low ionic strength. In $10 \mathrm{mM}$ Tris, $10 \mathrm{mM} \mathrm{NaCl}$, and $0.1 \mathrm{mM}$ EDTA at $\mathrm{pH} 8.0$, complete binding of the added protein was observed up to ratios of approximately $0.6 \mathrm{MBP} / \mathrm{DMPG} w / w$. Saturation of the binding was obtained at ratios above 1.5 MBP/DMPG $w / w$ added, and the saturation binding level corresponded to a protein:lipid ratio of $0.77: 1 \mathrm{w} / \mathrm{w}$, or 36 lipids per protein. Addition of MBP resulted in an instantaneous precipitation of the lipid-protein complexes due to rapid rates of vesicle aggregation and fusion (Young et al., 1982). The amount of protein binding was determined from lipid and protein determinations both on the pelleted complexes and on the supernatants, and was found to be consistent between the two. The isolated pellets were resuspended in the corresponding protein-free buffer, centrifuged, and then assayed for lipid and protein content. No protein was lost during such washes.

Phase Transition Behavior. The effect of MBP binding on the gel-to-liquid-crystalline phase transition of DMPG bilayers was examined by the following method. The outer splittings, $2 A_{\max }$, of the ESR spectra of 5-PGSL-labeled lipid-protein complexes were taken as empirical indicators of lipid chain mobility, and were measured as a function of temperature. The temperature dependence of $A_{\max }$ is shown in Figure 1 for different lipid:protein ratios. Very similar temperature dependences were also found for the effective order parameter, $S_{\text {eff }}$ (data not shown). DMPG exhibits a main disordering phase transition at $23^{\circ} \mathrm{C}$, which is gradually broadened with increasing protein:lipid ratio. At the saturating concentration of $2.5: 1 \mathrm{w} / \mathrm{w}$ added protein, the transition is completely abolished. The same result was obtained when the transition was monitored by a continuous temperature scan of the central line height of the 5-PGSL spin-label [for a description of the

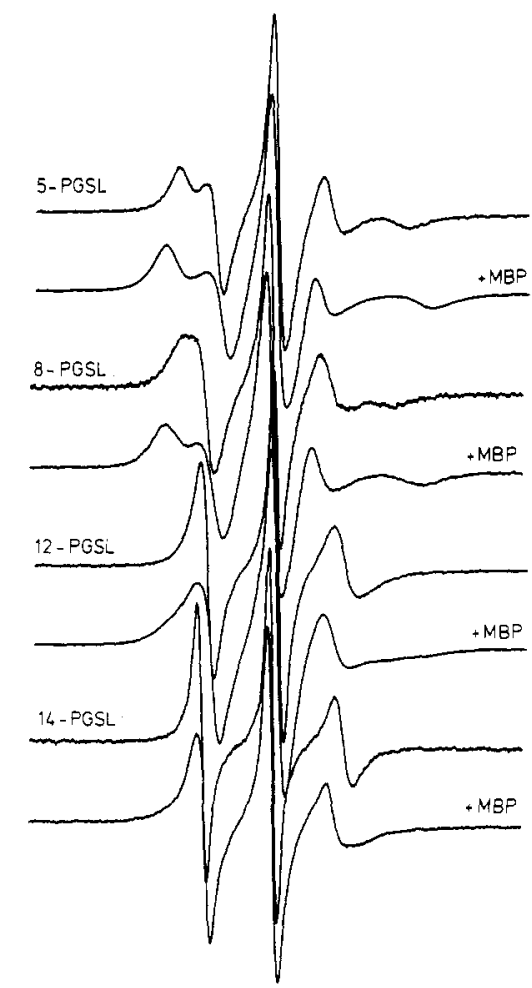

FIGURE 2: ESR spectra of phosphatidylglycerol spin-label positional isomers $n$-PGSL in DMPG dispersions $(10 \mathrm{mM}$ Tris, $10 \mathrm{mM} \mathrm{NaCl}$, and $0.1 \mathrm{mM}$ EDTA, pH 8.0) in the presence and absence of bovine MBP. The upper spectrum of each pair is from the lipid alone, and the lower spectrum is with a saturating amount of MBP bound (2.5:1 $\mathrm{w} / \mathrm{w}$ added MBP). Total spectral width is $100 \mathrm{G}$. $T=40^{\circ} \mathrm{C}$.

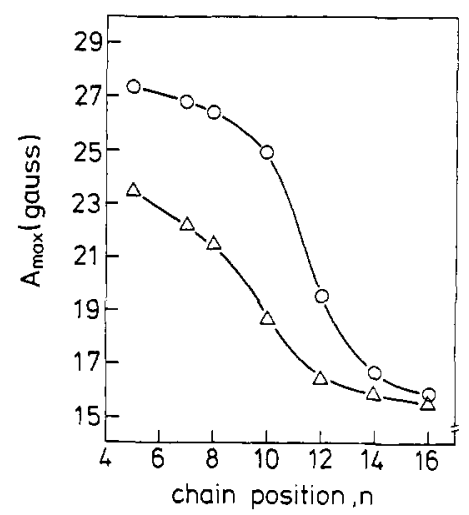

FIGURE 3: Outer hyperfine splittings $\left(A_{\max }\right)$ at $40^{\circ} \mathrm{C}$ as a function of nitroxide position, $n$, in the $s n-2$ chain for the $n$-PGSL phosphatidylglycerol positional isomers in DMPG dispersions. (O) In the presence of $2.5: 1 \mathrm{w} / \mathrm{w}$ added MBP; $(\Delta)$ in the absence of MBP.

method, see Watts et al. (1978)].

Chain Flexibility Gradient. The ESR spectra of the 5-, 7-, 10-, 12-, 14-, and 16-PGSL spin-labels are all sensitive to the presence of MBP. Figure 2 illustrates this effect, which is an increase in spectral anisotropy induced by the protein. The spectra of pure DMPG bilayers in the fluid phase $\left(30^{\circ} \mathrm{C}\right)$ exhibit axial symmetry and partial motional averaging. In each case, interaction with MBP gives rise to broader spectra with increased outer splittings, corresponding to an increased motional restriction of the spin-labeled chains for all the spin-label positional isomers examined. This is illustrated in Figure 3, where the outer hyperfine splittings of the PG spin-label positional isomers are plotted versus the chain position, for samples in the presence and absence of saturating quantities of protein. The characteristic flexibility gradient is seen both for pure DMPG bilayers and for lipid-protein complexes. In all the cases, both a spectral broadening and 


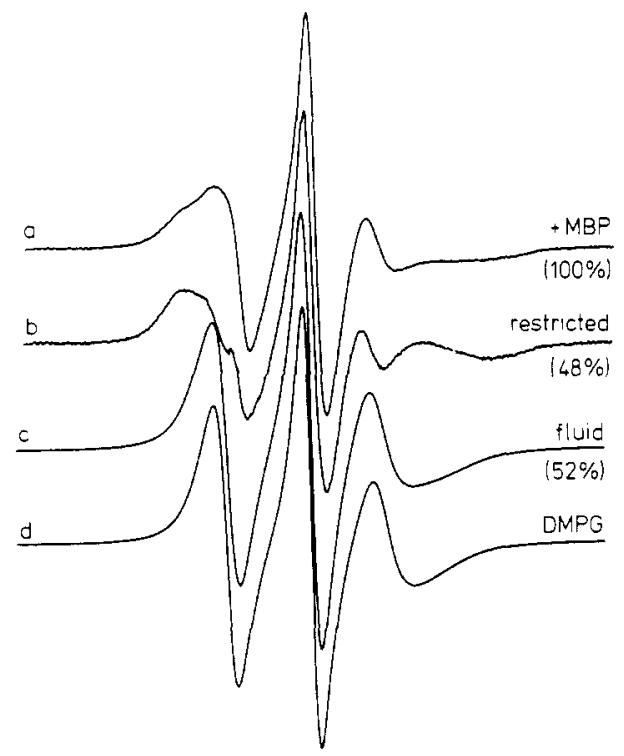

FIGURE 4: Spectral subtraction with the 12-PGSL phosphatidylglycerol spin-label. (a) Spectrum of a MBP-DMPG recombinant with lipid:protein ratio $=36: 1 \mathrm{~mol} / \mathrm{mol}$ recorded at $30^{\circ} \mathrm{C}$. (b) Difference spectrum obtained by subtracting the fluid spectrum $\mathrm{c}$ from spectrum a. (c) Fluid spectrum (pure DMPG small vesicles at $26^{\circ} \mathrm{C}$ ) used to obtain difference spectrum b. (d) Spectrum of the pure DMPG vesicles recorded at $30^{\circ} \mathrm{C}$. Total spectral width is $100 \mathrm{G}$.

an increase in the $A_{\max }$ values were induced by protein binding. Exactly similar differential profiles were obtained for the apparent order parameters, $S_{\text {eff }}$, as those shown for $A_{\max }$ in Figure 3. The spectral parameters of 5-PGSL were monitored as a function of protein binding (data not shown). $A_{\max }$ (and $\left.S_{\text {eff }}\right)$ increased from a value of $23.2 \mathrm{G}(0.44)$ for pure DMPG bilayers to a maximum value of $27.3 \mathrm{G}(0.68)$ with a saturating amount of protein bound. These changes paralleled the protein binding curve.

Two-Component Spectra. The spectra of the 12-PGSL and 14-PGSL lipid spin-labels in lipid-protein complexes consist of two components, in addition to exhibiting the generalized effects described above. One of the two components corresponds to the fluid bilayer lipid environment in the DMPGMBP recombinants. The other component is resolved in the outer wings of the spectrum and corresponds to spin-labeled lipid chains whose motion is more restricted than that of the fluid lipids. The outer hyperfine splitting of this component (deduced from difference spectra; see below) is approximately $53 \mathrm{G}$ for both 12 - and $14-\mathrm{PGSL}$ at $30^{\circ} \mathrm{C}$, which is significantly smaller than that obtained for the motionally restricted component observed with integral membrane proteins. No motionally restricted component was observed for the 16PGSL label, which indicates some chain length cutoff in the induction of this component by the MBP. In addition, no such second motionally restricted component was seen in the spectra of either 12- or 14-PGSL in complexes of DMPG with polylysine (data not shown), nor was it observed in complexes with cytochrome $c$ (Görrissen et al., 1986).

The two spectral components for the 12- and 14-PGSL labels are resolvable by digital subtractions [see, e.g., Marsh (1982)]. The results of such subtractions for the spectra of the 12-PGSL label in lipid-protein complexes are shown in Figure 4. The spectrum of the pure lipid recorded at a temperature which corresponds best to the fluid component is subtracted from the composite spectrum which was recorded at $30^{\circ} \mathrm{C}$ (Figure 4a). The matching fluid component in this case is the spectrum of 12-PGSL in DMPG bilayers at $26^{\circ} \mathrm{C}$ (Figure $4 c$ ). The difference spectrum (Figure $4 \mathrm{~b}$ ) is a sin-

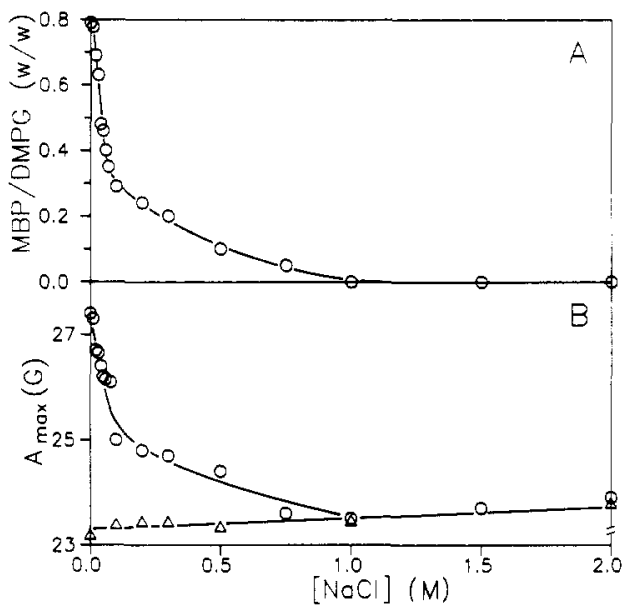

FIGURE 5: Salt dependence of (A) protein binding and (B) the outer hyperfine splitting, $A_{\max }$, of the 5-PGSL spin-label in DMPG at 40 ${ }^{\circ} \mathrm{C}$, in the presence $(0)$ and in the absence $(\Delta)$ of a saturating quantity $(2.5: 1 \mathrm{w} / \mathrm{w}$ added $)$ of MBP.

gle-component spectrum representing the motionally restricted spin-labeled lipid component [cf. Görrissen et al. (1986)]. The spectrum of DMPG alone at $30^{\circ} \mathrm{C}$, the same temperature as that of the recombinant in Figure 4a, is given for comparison in Figure 4d. The two-component spectra of 14-PGSL could also be resolved into a motionally restricted component and a fluid component, by digital spectral subtractions (data not shown). The quantitative results of these subtractions are found to be independent of the label position. The difference spectrum in Figure $4 \mathrm{~b}$ contains $48 \%$ of the total integrated intensity of the original composite spectrum (Figure 4a). This corresponds to approximately 18 lipids/MBP whose motion is restricted by the protein. Similar values for the fraction of motionally restricted lipid were also obtained from subtractions of spectra recorded at higher temperatures (up to $40^{\circ} \mathrm{C}$ for 12-PGSL). ${ }^{2}$

Ionic Strength and $p H$ Dependence. Large changes were observed both in protein binding and in the ESR spectra of the 5-PGSL label as a function of ionic strength. The dependence of MBP binding and of the outer hyperfine splitting of the 5-PGSL label on $\mathrm{NaCl}$ concentration is given in Figure 5. A steep decrease in the binding of MBP to DMPG bilayers was observed with increasing salt concentration. Protein binding was practically abolished at $\mathrm{NaCl}$ concentrations above $1 \mathrm{M}$. The corresponding spectral changes indicated a progressively decreasing perturbation of the lipid by the protein. $A_{\max }$ and $S_{\text {eff }}$ decrease with increasing salt concentration in a similar way to the protein binding, reaching a limiting value at $1 \mathrm{M} \mathrm{NaCl}$. Above this salt concentration, the $A_{\max }$ values are the same as those obtained for DMPG bilayers without protein. Both in the presence and in the absence of the protein, $A_{\max }$ increases slightly at high salt concentrations, presumably

${ }^{2}$ When the apparent splitting of the second motionally restricted component is much less than the rigid limit (unlike the case for integral proteins), it should be considered whether spectral line shapes such as that in Figure 4a can be described by a single-component model. The deciding point in this case is the consistency with the two-component model between different experimental spectra. In this work, similar proportions of the two components are found at different temperatures where at least the fluid component line shape changes. This is also true in the comparison between the spectra of 14-PGSL and 12-PGSL. In addition, different spin-labeled phospholipid species are found to display different selectivities in the proportion of the two components, whereas the individual component line shapes remain the same (Sankaram et al., 1989). Such consistencies cannot be reproduced by a single-component model. 


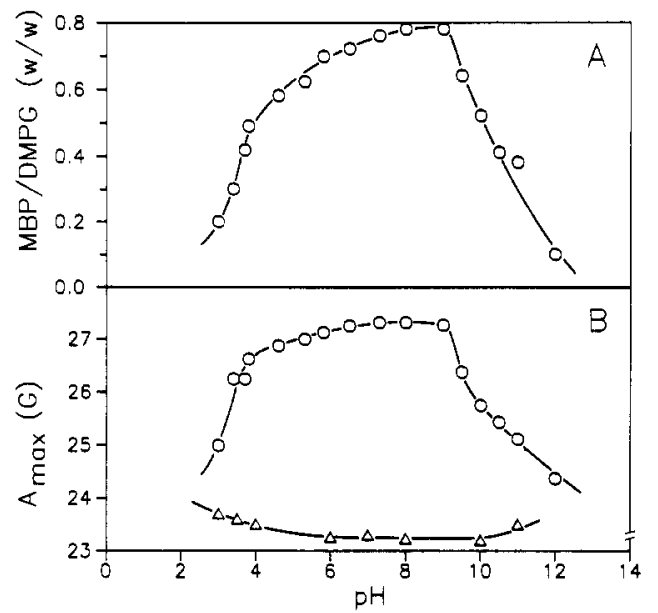

FIGURE 6: $\mathrm{pH}$ dependence of (A) the binding of MBP to DMPG dispersions in $10 \mathrm{mM}$ buffer plus $10 \mathrm{mM} \mathrm{NaCl}$ and (B) the outer hyperfine splitting, $A_{\max }$, of the 5-PGSL spin-label at $40^{\circ} \mathrm{C}$ in DMPG dispersions, in the presence $(O)$ and in the absence $(\Delta)$ of saturating amounts of MBP (2.5:1 w/w added).

due to binding of the salt to the phospholipid headgroups.

The interaction of MBP with DMPG bilayers is also dependent on $\mathrm{pH}$. The $\mathrm{pH}$ dependence of the protein binding, and of $A_{\max }$ for the 5-PGSL label, is shown in Figure 6. The protein binding changes only slightly in the $\mathrm{pH}$ range $9-5$. At pH values between 9 and 12 , a sharp decrease in protein binding and a concomitant decrease in $A_{\max }$ are observed. The isoelectric point of the myelin basic protein, $\mathrm{p} I=10.1$ (Yang et al., 1986), corresponds closely to the $\mathrm{p} K$ of this high-pH

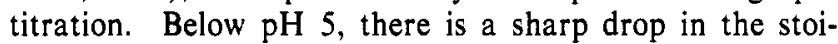
chiometry of protein/lipid bound. DMPG headgroups titrate in the $\mathrm{pH}$ range 3-3.5 (van Dijck et al., 1978; Watts et al., 1978); hence, the decrease in binding stoichiometry arises from titration of the DMPG headgroups which outweighs any effects of protonation of the protein side chains. Paralleling the protein binding behavior, $A_{\max }$ for 5-PGSL in lipid-protein complexes is approximately constant from $\mathrm{pH} 9$ down to $\mathrm{pH}$ 5 with a sharp drop at lower $\mathrm{pH}$ values.

\section{Discussion}

The interactions between MBP and negatively charged lipid bilayers formed from DMPG have been studied in this work by using spin-label ESR spectroscopy. Results are also presented on the $\mathrm{pH}$ dependence and ionic strength dependence of this interaction and are correlated with the results of chemical binding studies.

Binding Stoichiometry. Chemical binding assays of the lipid-protein complexes show a stoichiometry of approximately $36 \mathrm{~mol}$ of DMPG per mole of MBP bound at saturation. It might be expected that the binding stoichiometry of the MBP reflects in some way the shape and size of the protein, in addition to its distribution of positively charged residues. Hydrodynamic measurements and electron microscopy have indicated that the MBP in solution is a highly elongated molecule (Epand et al., 1974). The hydrodynamic measurements are consistent with a prolate ellipsoid of semiaxes $a=$ $86 \AA$ and $b=7.8 \AA$. Taking the area per lipid molecule of $60 \AA^{2}$ yields a stoichiometry of 35 lipids per protein for complete coverage of the bilayer surface. This value is in good agreement with the measured stoichiometry at saturation binding. Measurements on the stoichiometry of MBP complexed with bilayers composed of cerebroside sulfate $+40 \mathrm{~mol}$ $\%$ cholesterol yielded a rather similar value of 38 cerebroside sulfates per MBP (MacNaughtan et al., 1985). In terms of the charge stoichiometry, the MBP contains 31 positively charged residues, comprising 12 lysines and 19 arginines, which correlates quite well with the maximum number of negatively charged phospholipids associated with the protein at saturation binding. It is also interesting that the stoichiometry of MBP to negatively charged lipid in myelin lies within the same range as found in these model membrane experiments. For example, a value of 31 negatively charged lipids (phosphatidylserine and cerebroside sulfate) per MBP has been deduced for human myelin (Boggs et al., 1982a).

Bilayer Phase Transition. The gel-to-liquid-crystalline thermotropic phase transition of DMPG bilayers is affected by MBP binding. The transition is gradually broadened with increasing protein concentration consistent with the differential scanning calorimetric results on DMPG-MBP complexes (Boggs et al., 1982b) and a recent infrared spectroscopic investigation (Surewicz et al., 1987). In the $\mathrm{pH}$ range 5.9-8, the protein has been found to broaden the calorimetric transition and decrease $T_{\mathrm{t}}$ by $3.5-5^{\circ} \mathrm{C}$ while multiple transitions have been observed at low pH (Boggs et al., 1982b). The lipid:protein ratios of the complexes in this latter study ranged from 36.8 to $40.1 \mathrm{wt} \%(0.58-0.67 \mathrm{w} / \mathrm{w}$ MBP bound/DMPG). The extension to both higher and lower degrees of binding in the present study shows that the principal effect of MBP on the chain melting is a gradual reduction in the cooperativity of the transition with increasing amounts of protein bound (Figure 1). We observe a complete abolition of the chain melting transition when DMPG bilayers are saturated with MBP. Presumably, the intermolecular separation of the lipid molecules is then determined in part both by the distribution of positive charges on the protein and by the partial penetration of hydrophobic residues into the lipid. This restricts the freedom to undergo a cooperative phase transition.

Lipid Chain Flexibility Gradient. The protein-induced changes in the spectral parameters and line shapes, taken together with the results of digital spectral subtractions, suggest that binding of the basic protein has a general effect of motional restriction on acyl chain segmental motions. This is evidenced by the characteristic lipid chain flexibility gradient which is preserved on protein binding (see Figure 3). Previous results of Boggs and Moscarello (1978a), using a more limited range of stearic acid spin-label positional isomers, are essentially in accord with this finding. The outer hyperfine splittings and apparent order parameters of the different $n$-PGSL positional isomers are all increased, consistent with an increase in the lipid packing density induced by a surface interaction of the protein with the lipid headgroups. In addition, the MBP-DMPG complexes are best described as consisting of two populations of lipids which are motionally distinct on the spin-label ESR time scale (see Figure 4). One population corresponds to a motionally restricted population of lipids, which is similar in certain respects to that observed in the case of many integral membrane proteins (Marsh, 1985), and is therefore attributed to a direct interaction of the protein with the lipid chains. ${ }^{3}$ The other population is represented by the fluid lipid environment whose characteristic bilayer flexibility

\footnotetext{
${ }^{3}$ The motionally restricted lipid component is unlikely to be caused by chain interdigitation in the fluid phase, since the spectral splittings do not increase on going from the 12-position to the 14-position, and indeed no motionally restricted lipid component at all is observed with the 16-position label. A phase separation of the label may also be excluded, since spin-spin broadening is not observed (Figure $4 \mathrm{~b}$ ), the transition characteristics recorded by the spin-label show no evidence for such a phase separation (Figure 1), and measurements of the phase transition of the pure 12-PGSL label alone reveal that its transition temperature is not much higher than that of DMPG itself (data not shown).
} 
gradient is preserved but whose motion is uniformly hindered by the interaction of the protein at the bilayer surface. Although the motionally restricted component resolved by spectral subtractions is qualitatively similar to that obtained for many integral membrane proteins, there are certain important quantitative differences. First, the outer splittings of the resolved motionally restricted component are smaller ( 53 $G)$ than those observed for integral membrane proteins (typically $\geq 60 \mathrm{G}$ ). Second, a comparison of the line shapes suggests that the degree of motional restriction is less in the case of MBP-DMPG recombinants.

Protein Penetration. Two modes of interaction are therefore evidenced by the spectroscopic data. The first is a generalized decrease in the chain motion of all the lipids, resulting from the surface association of the protein. The second is a more direct perturbation of the lipid chain motion, most probably caused by a partial penetration of the protein into the hydrophobic region of the membrane. The extent of this penetration is difficult to assess, but the surface structure of the DMPG bilayer must be perturbed to such an extent that labels at the C-14 atom position of the lipid chain come into more direct contact with the protein. However, as mentioned under Results, no such interaction is seen at the C-16 atom position, indicating that the penetration or perturbation is only partial in nature. Examination of the disposition of hydrophobic residues in the sequence of the MBP (Weiss, 1985) reveals that there are no extensive hydrophobic sequences which could constitute membrane-spanning helices. Therefore, the interaction with the hydrophobic part of the lipid bilayer is, as noted above, rather different from that found with integral membrane proteins, and must be of a more superficial nature. In these respects, the mode of lipid-protein association (and the spectral effects) is very similar to that observed previously for apocytochrome $c$ (Görrissen et al., 1986). In the latter case, it was suggested that the membrane penetration might be associated with the translocation of the apoprotein. In the case of the MBP, it may be that the membrane penetration plays a role in stabilizing the structure of the myelin sheath and/or in interactions between the MBP and the myelin proteolipid apoprotein.

The myelin basic protein differs from apocytochrome $c$ with respect to the relative amount of lipid which is motionally restricted. The MBP with molecular weight of $18.4 \mathrm{~K}$ restricts approximately 18 lipids/protein, whereas apocytochrome $c$ with a molecular weight of $11.5 \mathrm{~K}$ restricts a maximum of $4-5$ lipids/protein and only 2 lipids/protein at saturation protein binding (Görrissen et al., 1986). This is in line with the idea that the penetration of the MBP plays a structural role in the myelin sheath, whereas penetration of apocytochrome $c$ has only a transient function in protein import. The number of lipids motionally restricted by MBP is equivalent to that which could be accommodated around four cylinders of diameter ca. $10 \AA$, if they were to penetrate less than halfway into the membrane. This implies that on the order of maximally 40 of the total 170 residues of the MBP may penetrate into the membrane if they are in a helical arrangement, or approximately 32 residues if they arranged in a $\beta$-sheet structure [cf. Surewicz et al. (1987)]. For amphipathic helices oriented parallel to the membrane surface, the total helix length would be approximately $68 \AA$, corresponding to ca 45 residues. In each case, this constitutes a significant fraction of the total mass of the protein.

Other lines of evidence exist for the partial membrane penetration of the MBP. Proteolytic digestion of membrane-bound MBP has shown that specific regions of the molecule are protected by the membrane (London et al., 1973). Calorimetric measurements have shown that the MBP induces a large decrease in the enthalpy of the gel-to-liquid-crystalline phase transition of DPPG bilayers (Boggs \& Moscarello, 1978b; Papahadjopoulos et al., 1975). Additionally, the hysteresis in the phase transition induced by MBP can be interpreted in terms of a penetration of the protein into the bilayer in the fluid state (Boggs \& Moscarello, 1978a). Monolayer studies have indicated a substantial protein-induced expansion in area of closely packed lipid monolayers (Papahadjopoulos et al., 1975). An enhanced hydrophobic photolabeling of MBP observed in the presence of negatively charged lipid vesicles is also consistent with a partial penetration of the MBP into the membrane (Boggs et al., 1988). ESR studies on complexes of MBP with various negatively charged phospholipids (Boggs et al., 1980; Stollery et al., 1980) and with synthetic cerebroside sulfates (Boggs et al., 1985) using iodoacetamide-labeled protein have also been interpreted in terms of a hydrophobic interaction of the MBP with the lipid. The ESR evidence presented here directly identifies the motionally restricted lipid component induced by the protein.

Ionic Strength and $p H$ Dependence. An obligatory role is played by electrostatic interactions in the binding of MBP to DMPG bilayers. The binding of the protein decreases with increasing ionic strength, as the charged lipid headgroups are screened by counterions. The ionic strength dependence is qualitatively that expected from Gouy-Chapman electrostatic double-layer theory [cf. Cevc and Marsh (1987)] and closely resembles the experimentally determined surface potential of DMPG bilayers as a function of salt concentration (Cevc et al., 1980). At high salt concentrations, the membranes are totally depleted of the protein (Figure 5). Further, in agreement with the results of Papahadjopoulos et al. (1975), we find no binding of MBP to DMPC vesicles. These results indicate that possible hydrophobic interactions are insufficient alone to cause binding of the MBP to lipid membranes. Although they may add to the total net binding energy, the electrostatic interaction is a necessary prerequisite.

Binding of the MBP to DMPG bilayers is sensitive to $\mathrm{pH}$. On titration to $\mathrm{pH}$ values close to the isoelectric point $(\mathrm{p} I=$ 10.1) of the protein, the binding decreases, presumably due to deprotonation of the basic amino acid residues. This reduction in binding is accompanied by a concomitant decrease in the outer hyperfine splittings of the phospholipid labels. At $\mathrm{pH}$ values below 4.5 , the protein/lipid stoichiometry of the binding is reduced, with an accompanying decrease in the $A_{\max }$ values. This low-pH titration corresponds to the protonation of the DMPG headgroups (van Dijck et al., 1978; Watts et al., 1978). The decrease in negative charge on the membrane surface due to this titration leads to a reduced protein binding. Protonation of the amino acid side chains of the protein in the low-pH region would tend to strengthen the electrostatic attraction, but it is clear from Figure 6 that the effect of the reduced bilayer surface charge dominates in this case.

In conclusion, the salt and $\mathrm{pH}$ dependences indicate that an electrostatic interaction is obligatory for the binding of the myelin basic protein to negatively charged lipid bilayers. However, the spin-label results also provide evidence for a more direct interaction of the protein with the lipid chains, in addition to surface binding to the bilayer. This hydrophobic interaction may be necessary for stabilizing the compact structure of the myelin sheath.

\section{ACKNOWLEDGMENTS}

We thank B. Angerstein and S. Chatterjee for expert technical assistance. 
Registry No. DMPG, 61361-72-6; DMPC, 18194-24-6.

\section{REFERENCES}

Boggs, J. M., \& Moscarello, M. A. (1978a) J. Membr. Biol. 39, 75-96.

Boggs, J. M., \& Moscarello, M. A. (1978b) Biochim. Biophys. Acta 515, 1-21.

Boggs, J. M., Moscarello, M. A., \& Papahadjopoulos, D. (1982a) in Lipid-Protein Interactions (Jost, P. C., \& Griffith, O. H., Eds.) Vol. 2, pp 1-51, Wiley, New York.

Boggs, J. M., Stamp, D., \& Moscarello, M. A. (1982b) Biochemistry 21, 1208-1214.

Boggs, J. M., Rangaraj, G., \& Koshy, K. M. (1988) Biochim. Biophys. Acta 937, 1-9.

Braun, P. E. (1984) in Myelin (Morell, P., Ed.) pp 97-116, Plenum Press, New York.

Cevc, G., \& Marsh, D. (1987) Phospholipid Bilayers. Physical Principles and Models, 442 pp, Wiley-Interscience, New York.

Cevc, G., Watts, A., \& Marsh, D. (1980) FEBS Lett. 120, 267-270.

Chao, I. P., \& Einstein, E. R. (1970) J. Neurochem. 17, $1121-1132$

Comfurius, P., \& Zwaal, R. F. A. (1977) Biochim. Biophys. Acta 488, 36-42.

Diebler, G. E., Martenson, R. E., \& Kies, M. W. (1972) Prog. Biophys. Biochem. 2, 139-165.

Eibl, H., \& Lands, W. E. M. (1969) Anal. Biochem. 30, 51-57.

Epand, R. M., Moscarello, M. A., Zierenberg, B., \& Vail, W. J. (1974) Biochemistry 13, 1264-1267.

Eylar, E. H., \& Thompson, M. (1969) Arch. Biochem. Biophys. 129, 469-479.

Eylar, E. H., Caccam, J., Jackson, J., Westall, F., \& Robinson, A. B. (1970) Science 168, 1220.

Görrissen, H., Marsh, D., Rietveld, A., \& de Kruijff, B. (1986) Biochemistry 25, 2904-2910.

Horvāth, L. I., Brophy, P. J., \& Marsh, D. (1988) Biochemistry 27, 5296-5304.

Jost, P. C., Libertini, L. J., Hebert, V. C., \& Griffith, O. H. (1971) J. Mol. Biol. 59, 77-98.

Kies, M. W., Murphy, J. B., \& Alvord, E. C., Jr. (1961) in Chemical Pathology of the Nervous System (Folch-Pi, J., Ed.) pp 197-206, Pergamon Press, London.

Laatsch, R. H., Kies, M. W., Gordon, S., \& Alvord, E. C., Jr. (1962) J. Exp. Med. 115, 777-788.
Laemmli, U. K. (1970) Nature (London) 227, 680-685.

Lange, A., Marsh, D., Wassmer, K.-H., Meier, P., \& Kothe, G. (1985) Biochemistry 24, 4383-4392.

Liebes, L. F., Zand, R., \& Phillips, W. D. (1975) Biochim. Biophys. Acta 405, 27-39.

London, Y., Demel, R. A., Geurts van Kessel, W. S. M., Vossenberg, F. G. A., \& van Deenen, L. L. M. (1973) Biochim. Biophys. Acta 311, 520-530.

Lowry, O. H., Rosebrough, N. J., Farr, A. C., \& Randall, R. J. (1951) J. Biol. Chem. 193, 265-275.

MacNaughtan, W., Snook, K. A., Caspi, E., \& Franks, N. P. (1985) Biochim. Biophys. Acta 818, 132-148.

Marsh, D. (1981) in Membrane Spectroscopy (Grell, E., Ed.) pp 51-142, Springer-Verlag, Berlin.

Marsh, D. (1982) Tech. Life Sci.: Biochem. B4/II, B426/ $1-B 426 / 44$.

Marsh, D. (1985) in Progress in Protein-Lipid Interactions (Watts, A., \& de Pont, J. J. H. H. M., Eds.) pp 143-172, Elsevier, Amsterdam.

Marsh, D. (1986) in Supramolecular Structure \& Function (Pifat-Mrzljak, G., Ed.) pp 48-62, Springer-Verlag, Berlin.

Marsh, D., \& Watts, A. (1982) in Lipid-Protein Interactions (Jost, P. C., \& Griffith, O. H., Eds.) Vol. 2, pp 53-126, Wiley-Interscience, New York.

Moscarello, M. A., Katona, E., Neumann, A. W., \& Epand, R. M. (1974) Biophys. Chem. 2, 290-295.

Palmer, F. B., \& Dawson, R. M. C. (1969) Biochem. J. 111, 629-636.

Papahadjopoulos, D., Moscarello, M., Eylar, E. H., \& Isac, T. (1975) Biochim. Biophys. Acta 401, 317-335.

Sankaram, M. B., Brophy, P. J., \& Marsh, D. (1989) Biochemistry 28, 9699-9707.

Stone, A. L., Park, J. Y., \& Martenson, R. E. (1985) Biochemistry 24, 6666-6673.

Surewicz, W. K., Moscarello, M. A., \& Mantsch, H. H. (1987) Biochemistry 26, 3881-3886.

van Dijck, P. W. M., de Kruijff, B., Verkleij, A. J., van Deenen, L. L. M., \& de Gier, J. (1978) Biochim. Biophys. Acta 512, 84-96.

Watts, A., Harlos, K., Maschke, W., \& Marsh, D. (1978) Biochim. Biophys. Acta 510, 63-74.

Weiss, J. (1985) J. Neurochem. 44, 163-170.

Yang, J. C., Changtung Chan, P., Fujitaki, J. M., Chui, K. C., \& Smith, R. A. (1986) Biochemistry 25, 2677-2681.

Young, P. R., Vaconte, D. A., \& Snyder, W. R. (1982) J. Am. Chem. Soc. 104, 7287-7291. 